\title{
Clinical Trial Unblinding Documentation
}

National Cancer Institute

\section{Source}

National Cancer Institute. Clinical Trial Unblinding Documentation. NCI Thesaurus. Code C115498.

Records pertaining to the reversal of randomization code to reveal a specific subject's trial group allocation data. 